\title{
Innovations for the new year
}

\section{This week's issue looks at new laboratory products to make research safer, faster and easier,} including a robotic arm, a see-through incubator and a pocket photometer.

BETHESDA Research Laboratories has a new electrophoresis system for miniature gels measuring $5.7 \times 8.3 \mathrm{~cm}$ (Reader Service No. 100). The Horizon 58 unit features a slide-out gel-casting dam that eliminates the use of tape, and a thick liftout plastic gel deck that ensures warp-free casting without precooling the agarose.

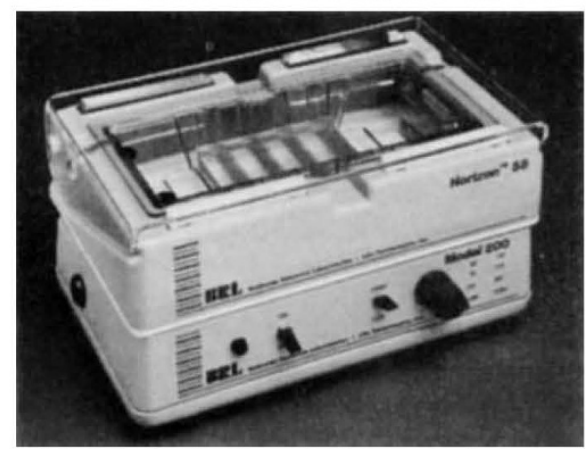

BRL's mini-gel unit stacks for convenience.

The buffer tray holds $100 \mathrm{ml}$ of buffer, and can be removed easily for buffer disposal. The gel casting unit includes gel-casting deck, dams, buffer tray, base unit, 8- and 14-well combs, and power cords. The unit can be stacked on top of the model 200 power supply, sold separately, to save space on the lab bench.

The American Type Culture Collection has announced its series of workshops for 1988 (Reader Service No. 101). Topics for January through June include: an update on hybridomas and monoclonal antibodies, recombinant DNA technology, freezing and quality control of cell cultures and hybridomas, freezing and freeze-drying of microorganisms, and gas chromatographic analysis of cellular fatty acids for bacterial identification. The laboratory-intensive workshops last from two to five days, and count for up to three CEU credits. In April, the ATCC is also sponsoring a biotechnology patent conference, including discussions of national and international patenting requirements, and the current status of patenting and depositing animal varieties.

An environmental cabinet for breeding laboratory animals is available from Equipements Scientifiques \& Industriels (Reader Service No. 102). The model E110S has a self-contained air flow system that comes in either a positive or negative pressure configuration. The positive pressure cabinet maintains a germ-free atmosphere for animals who require protection against pathogens, while the negative pressure cabinet can be used to protect the laboratory environment from pathogens carried by animals inside the cabinet.
Both models have a prefilter that screens out 90 per cent of particles over $5 \mu \mathrm{m}$, and an absolute filter to remove 99 per cent of $0.3-5.0 \mu \mathrm{m}$ particles. Each cabinet holds about 16 medium-sized cages, and has four separate compartments and eight doors.

DR Lange has a pocket photometer for water analysis, the LASA Aqua (Reader Service No. 103). The unit has 14 program filters for the analysis of ammonium, cadmium, calcium, chloride, chlorine, chlorine dioxide, chromium, copper, cyanide, fluoride, formaldehyde, hexacyanoferrates, iron, magnesium, nickel, nitrate, nitrite, ozone, phenols, phosphate, potassium, silver, sulphate, zinc and water-hardness. The appropriate filters needed to generate the wavelength suitable for the analysis of a particular ion

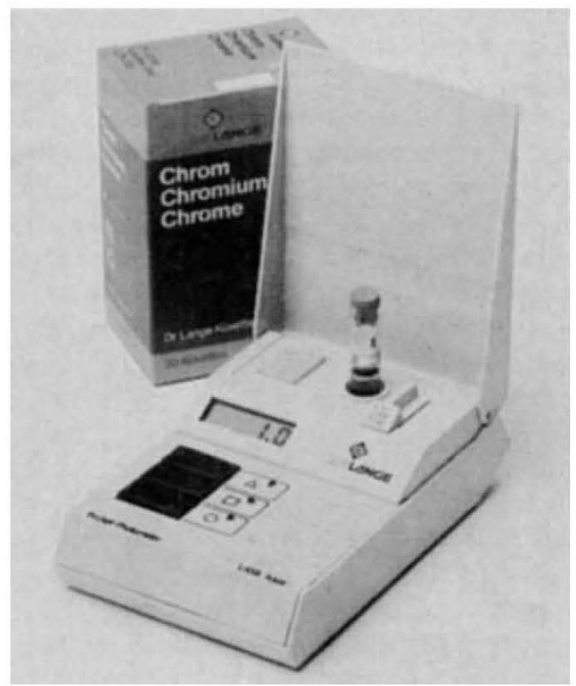

The DR Lange photometer fits in a pocket.

are put in place when one of the three operation buttons are pressed. The LASA Aqua is automatically calibrated, so no zero-adjustment using blank cuvettes is required.

A new brochure from Perkin-Elmer describes the MasterLab robot system for sample preparation and analysis (Reader Service No. 104). The free brochure outlines the capabilities of the MasterLab core system, which consists of a hingedarm robot designed to emulate the human arm, a system controller, and PerkinElmer robot language. The MasterLab robot is designed for use in a chemical or pharmaceutical laboratory. It features a linear transport belt to allow the robot to move, and interchangeable tactile-sensing 'fingers' for grasping objects ranging from test tubes to microtitre plates. Liquid chromatographs, Mettler titrators,
UV-visible spectrophotometers and other instruments may be interfaced to the MasterLab system, and the robot can be programmed to perform sample injection. The robot can also perform weighing steps, pipetting, centrifugation, and mixing, using a vortex mixer or rotating sample racks.

For keeping an eye on samples under incubation, Stuart Scientific sells an acrylic 60-litre incubator, the model SI60

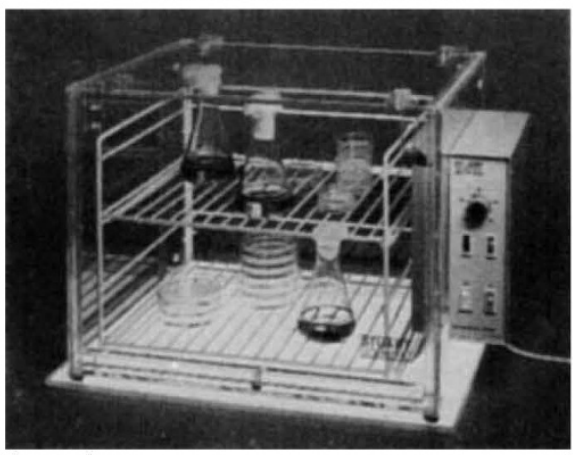

See what you grow with Stuart Scientific's incubator.

(Reader Service No. 105). The incubator opens at the top, front and base, and the wire rack may be removed for incubating entire instruments. For working with anaerobes, the unit is available with glove ports and inlets for introducing cables or gases. With the addition of a salt-tray humidifier, the incubator becomes a noninjection humidity chamber. Stuart Scientific says fan convection within the unit ensures uniform humidity and temperature fluctuation of less than $0.1^{\circ} \mathrm{C}$.

ICN Biomedicals has a brochure devoted to animal research diets (Reader Service No. 106). The free brochure lists information on the 100 standard diets and the over 10,000 custom formulations sold by the company. The diets come in three pellet sizes, and are colour-coded to ease control of administration.

Laboratory personnel can be shielded from beta radiation from liquid radioactive waste with Rad-Can beta shields from Research Products International (Reader Service No. 107). The acrylic boxes come in two sizes: the $\$ 75$ (US) Rad-Can II holds a one gallon bottle, and the $\$ 79$ (US) Rad-Can III accepts a twogallon carboy.

These notes are compiled by Carol Ezzell from information provided by the manufacturers. To obtain further details about these products, use the reader service card bound inside the journal. Prices quoted are sometimes nominal and apply only within the country indicated. 